\title{
Flow Structures of Gaseous Jet Injected into Liquid for Underwater Propulsion
}

\author{
Jianing Tang ${ }^{1}$, Shipeng $\mathrm{Li}^{2}$ and Ningfei Wang ${ }^{3}$ \\ Beijing Institute of Technology, Beijing, 100081, China \\ Yingjie $\mathrm{Wei}^{4}$ \\ Harbin Institute of Technology, Harbin, 150001, China \\ Wei Shyy ${ }^{5}$ \\ University of Michigan, Ann Arbor, MI, 48109, U.S.A.
}

\begin{abstract}
Gaseous jet injected into liquid typically occurs in underwater propulsion. The flow is unsteady and turbulent; the high density ratio between water and gas can induce many complicated phenomena. To investigate the underwater propulsion performance, the analytical gas bubble model which has been widely used to predict the thrust of underwater engine is summarized. In this model, water is assumed to form a conservative system, so that the work done on the water by the gas is equal to the sum of the kinetic and potential energies stored in water. To assess the validity of this approximate model, the detailed Navier-Stokes flow computations were made to elucidate flow structures of the gasliquid interactions and to evaluate their effect on the propulsion system. Specifically, multiphase unsteady flows were simulated using the Volume of Fluid model; fluid compressibility, viscosity, and energy transfer were taken into account. The results show that phenomena including expansion, necking/breaking, and back-attack were highlighted in the jet process, which cannot be described by the analytical model. Our analysis indicates that the back pressure in underwater propulsion is much different from the ambient pressure and can significantly affect the propulsion performance. When gas is fully expanded, the back pressure can be lower than the ambient pressure, which results in a decrease in total thrust. However, when the necking/breaking appears, the back pressure will jump to a high value, which affects the mass flow rate through the nozzle, the velocity and the pressure at the nozzle exit. Thus, the total thrust in underwater propulsion is not only determined by the nozzle geometry but also by the flow structures and associated pressure distributions.
\end{abstract}

\section{Nomenclature}

$A_{t} \quad=$ Area of nozzle throat

$A_{e} \quad=$ Area of nozzle exit

$A_{s} \quad=$ Area of cross-section of propulsion system

$d_{t} \quad=$ Diameter of nozzle throat

$d_{s} \quad=$ Diameter of cross-section of propulsion system

$D \quad=$ Diameter of nozzle exit

$E_{w, k} \quad=$ Kinetic energy of water

$E_{w, p} \quad=$ Potential energy of water

\footnotetext{
${ }^{1}$ Ph.D. candidate. Beijing Institute of Technology, Department of Aerospace Engineering, tjn1216@gmail.com, Member AIAA.

${ }^{2}$ Associate Professor. Beijing Institute of Technology, Department of Aerospace Engineering, lsp@bit.edu.cn, Member AIAA.

${ }^{3}$ Professor. Beijing Institute of Technology, Department of Aerospace Engineering, wangningfei@bit.edu.cn, Member AIAA.

${ }^{4}$ Associate Professor. Harbin Institute of Technology, School of Astronautics, weiyingjie@gmail.com, Member AIAA.

${ }^{5}$ Clarence L. "Kelly" Johnson Collegiate Professor and Chair, University of Michigan, Department of Aerospace Engineering,weishyy@umich.edu, Fellow AIAA.
} 


\begin{tabular}{|c|c|c|}
\hline$E_{b}$ & $=$ & Energy of gas bubble \\
\hline$F$ & $=$ & Thrust \\
\hline$F^{*}$ & $=$ & Normalized thrust \\
\hline$k_{t}$ & $=$ & Turbulent thermal conductivity \\
\hline$k_{g}$ & $=$ & Thermal conductivity of gas \\
\hline$k_{w}$ & $=$ & Thermal conductivity of water \\
\hline$k_{\text {eff }}$ & $=$ & Effective thermal conductivity, $k_{e f f}=k+k$ \\
\hline$\dot{m}$ & $=$ & Mass flow rate \\
\hline$M$ & $=$ & Mach number \\
\hline$P$ & $=$ & Designed nozzle exit pressure \\
\hline$p_{e}$ & $=$ & Pressure at nozzle exit \\
\hline$p_{b}$ & $=$ & Pressure in gas bubble \\
\hline$p_{B}$ & $=$ & Back pressure \\
\hline$p_{0}$ & $=$ & Stagnant pressure \\
\hline$p_{1}$ & $=$ & Atmosphere pressure \\
\hline$p_{a}$ & $=$ & Ambient pressure \\
\hline$p^{*}$ & $=$ & Normalized pressure \\
\hline$P_{r, g}$ & $=$ & Prandtl number of gas \\
\hline$P_{r, w}$ & $=$ & Prandtl number of water \\
\hline Re & $=$ & Reynolds number \\
\hline$R_{g}$ & $=$ & Gas constant \\
\hline$T_{0}$ & $=$ & Stagnant temperature \\
\hline$v_{\mathrm{e}}$ & $=$ & Velocity at nozzle exit \\
\hline$U^{*}$ & $=$ & Normalized Axial-velocity \\
\hline$U$ & $=$ & Designed nozzle exit velocity \\
\hline$\rho_{w}$ & $=$ & Density of water \\
\hline$\rho_{g}$ & $=$ & Density of gas \\
\hline$\rho_{m}$ & $=$ & Density of mixture \\
\hline$\mu_{w}$ & $=$ & Dynamic viscosity of water \\
\hline$\mu_{g}$ & $=$ & Dynamic viscosity of gas \\
\hline$\mu_{m}$ & $=$ & Dynamic viscosity of mixture \\
\hline$\alpha_{w}$ & $=$ & Volume fraction of water \\
\hline$\alpha_{g}$ & $=$ & Volume fraction of gas \\
\hline$\gamma$ & $=$ & Specific heats of gas \\
\hline
\end{tabular}

\section{Introduction}

$\mathrm{T}$

The flow process of gaseous jet injected into liquid is unsteady and turbulent. It is encountered in a variety of engineering applications such as direct-contact condensers, metallurgical processes, underwater cutting, underwater propulsion, and so on. These wide-range applications of such flows have received much attention.

So far, a considerable amount of effort has been directed towards measurement, observation, and modeling methods of the flow structures for submerged gaseous jets. A low speed gas jet usually turns into bubbly flow ${ }^{1}$, which can be well described by a theoretical model ${ }^{2}$. For a high speed jet, He et al. ${ }^{3}$ calculated the flow field by using the Level Set method. George et al. ${ }^{4}$ examined the dynamics of annular gas-liquid two-phase jets by means of direct numerical simulation. Cao et al. ${ }^{5}$ analyzed the dynamic characteristics of underwater supersonic gas jets at different depths, with different nozzle-expansion ratios. Lindau et al. ${ }^{6}$ developed an algorithm for modeling compressible phenomena in multiphase, reacting flows, and used the formulation to calculate a hypothetical high-speed supercavitatingvehicle propulsion plum. Surin et $\mathrm{al}^{7}{ }^{7}$ conducted an experimental study of the discharge of a gas jet from a nozzle and its development in a liquid. The study focused on the dynamics of gas jet in a liquid, the structure of the region of interaction, and the regimes of discharge from the submerged nozzle with different degrees of gas assimilation. Koria ${ }^{8}$ presented a simple structure model and calculated the non-buoyant jet length. He concluded that for injection velocities of $\mathrm{M}<1$, the gas jet disintegrates in the form of bubbles very close to the gas injecting orifice, and a bubble column forms in a metal bath. For $\mathrm{M}>1$, the length of the non-buoyant jet in the bath was appreciable, and the jet later disintegrated into bubbles. Qi et al. ${ }^{9}$ and Loth and Faeth ${ }^{10}$ provided strong evidence to show the presence of a shock-wave-containing expansion region for air jets in water. Studies on shock waves have also focused on their 
movement ${ }^{11}$, reflection ${ }^{12-15}$, and influence of the confined boundary on their structure. For example, Abate and Shyy ${ }^{13}$ investigated the gas dynamic phenomenon of a normal shock wave within a tube undergoing a sudden area expansion. They focused on multiple gaseous mechanisms associated with highly transient flow and diffraction that give rise to turbulent, compressible, vortical flows, including shock-strain rate interaction, baroclinic effect, vorticity generation, and different aspects of viscous dissipation.

Numerous studies indicate that the jet-induced effect is an important characteristic in submerged gas jet process. For example, Dai et al. ${ }^{16}$ described an experimental investigation of the flow pattern and hydrodynamic effect of underwater gas jets from supersonic and sonic nozzles operated in both perfect and imperfect expansion conditions. The obtained results showed that high-speed gas jets in still water induced large pressure pulsations upstream of the nozzle exit. The presence of shock-cell structures in the over- and under-expanded jets led to an increase in the intensity of the jet-induced hydrodynamic pressure. Shi et al. ${ }^{17,18}$ indicated that the process of supersonic air jets ejecting in water is accompanied by strong flow oscillation, which is induced by jet process and related to shock waves reflecting in the gas phase. Wang et al. ${ }^{19}$ presented an experimental study on the back-attack phenomenon in underwater supersonic gas jets. The development of back-attack phenomenon was visualized using a digital camera. The fluctuating pressure distributions in the near field of the jet were obtained by a row of probes. The experimental findings indicated that in the region two diameters away from the nozzle exit downstream, the distortion of the gas jet induced a strong pressure pulse. Thus, for underwater propulsion, jet-induced effect is likely to be the distinguishing feature as compared to aerial propulsion.

An analytical gas bubble model which is based on the Rayleigh-Plesset equation has been widely used to predict the performance of underwater engines because it approximately describes the inertia effect of water. Rogers ${ }^{20}$ used this model to predict the pressure during the ignition process; Richard ${ }^{21}$ used this model to determine the minimum starting transient for a given allowable pressure in an underwater engine; Wang et al. ${ }^{22}$ improved and used this model to study the flow status in the Laval nozzle under water; and Zhang et al. ${ }^{23}$ also used this model to study the engine's thrust at the initial transient. However, as indicated by Shi et al. ${ }^{17,}{ }^{18}$, complicated phenomena such as changes in pressure are involved in the injection of a submerged supersonic gas jet. The single bubble model is a low order approximation of the complex jet structure.

In the present work, we aim to study the flow structures and phenomena involved in submerged supersonic gas jets; to investigate the characteristics of jet-induced effects; and to evaluate the influences on underwater propulsion performance so that a better understanding of underwater propulsion can be achieved.

\section{Analytical gas bubble model}

In this analytical gas bubble model, gas is represented as a single bubble which can expand in an infinite water region; the expansion velocity is in the radial direction and equal to $d R / d t$; the pressure and density distributions in the bubble are set to be uniform. Water is assumed to form a conservative system, so the work done on the water by the gas is equal to the kinetic energy plus the potential energy stored in water; the total energy exported from the nozzle is equal to the total energy stored in gas bubble and water. The relationship among water, gas bubble and nozzle was summarized by Wang et $_{\text {al. }}{ }^{22}$ as follows:

$$
\begin{gathered}
p_{b}=\rho_{w}\left[\frac{3}{2}\left(\frac{d R}{d t}\right)^{2}+R \frac{d^{2} R}{d t^{2}}\right]+\rho_{w} g H+p_{1} \\
\frac{d p_{b}}{d t} \cdot \frac{4}{3} \pi R^{3}+\gamma p_{b} \cdot 4 \pi R^{2} \frac{d R}{d t}=\gamma R_{g} T_{0} \dot{m}
\end{gathered}
$$

where $R$ is the radius of gas bubble, $p_{b}$ is the pressure in gas bubble, $\rho_{w}$ is the density of water, $T_{0}$ is stagnant temperature, $H$ is the depth of location, $p_{l}$ is the atmosphere pressure, $\gamma$ is the ratio of specific heats of gas, $R_{g}$ is the gas constant, and $\dot{m}$ is the mass flow rate. On the assumption that the gas flow in the nozzle is one-dimensional and the pressure in the gas bubble is equal to the back pressure, the mass flow rate can be controlled using the following 3 conditions: 


$$
\dot{m}\left\{\begin{array}{lr}
\frac{p_{0} A_{t}}{\sqrt{R_{g} T_{0}}} \cdot\left(\frac{2}{\gamma+1}\right)^{\frac{\gamma+1}{2(\gamma-1)}} \sqrt{\gamma} & p_{b} \leq p_{b}^{*} \\
\frac{p_{0} A_{e}}{\sqrt{R_{g} T_{0}}} \cdot\left(\frac{p_{b}}{p_{0}}\right)^{\frac{1}{\gamma}}\left\{\frac{2 \gamma}{\gamma-1}\left[1-\left(\frac{p_{b}}{p_{0}}\right)^{\frac{\gamma-1}{\gamma}}\right]\right\}^{\frac{1}{2}} & p_{b}^{*}<p_{b}<p_{0} \\
0 & p_{b} \geq p_{0}
\end{array}\right.
$$

Here, $p^{*}{ }_{b}$ is the critical pressure and $p_{0}$ is the stagnant pressure. $p_{b}, \dot{m}$ and $R$ can be obtained from the nonlinear differential equation set (1)-(3). Numerical solutions were matched in time by using the Runge-Kutta method of second order accuracy. The initial conditions are as follows: $\left.R\right|_{t=0}=0.01, d R /\left.d t\right|_{t=0}=0,\left.p_{b}\right|_{t=0}=p_{1}+\rho_{w} g H$.

\section{Navier-Stokes flow computation}

\section{A. Governing equation}

The set of governing equations consists of the conservative form of the Navier-Stokes equations, the original $k-\varepsilon$ two-equation turbulence closure, and a transport equation for the gas volume fraction. The continuity, momentum, energy and VOF model equations are given below.

$$
\begin{gathered}
\frac{\partial \rho_{m}}{\partial t}+\frac{\partial\left(\rho_{m} u_{i}\right)}{\partial x_{i}}=0 \\
\frac{\partial\left(\rho_{m} u_{i}\right)}{\partial t}+\frac{\partial\left(\rho_{m} u_{i} u_{j}\right)}{\partial x_{j}}=-\frac{\partial p}{\partial x_{i}}+\frac{\partial}{\partial x_{j}}\left[\mu_{m}\left(\frac{\partial u_{i}}{\partial x_{j}}+\frac{\partial u_{j}}{\partial x_{i}}\right)\right]+F_{i} \\
\frac{\partial}{\partial t} \sum_{k=w, g}\left(\alpha_{k} \rho_{k} E_{k}\right)+\frac{\partial}{\partial x_{j}} u_{j} \sum_{k=w, g}\left[\alpha_{k}\left(\rho_{k} E_{k}+p\right)\right]=\frac{\partial}{\partial x_{j}}\left(k_{e f f} \frac{\partial T}{\partial x_{j}}\right) \\
\frac{\partial}{\partial t}\left(\alpha_{g} \rho_{g}\right)+\frac{\partial}{\partial x_{i}}\left(\alpha_{g} \rho_{g} u_{i}\right)=0
\end{gathered}
$$

The mixture property, $\varphi_{m}$ can be expressed as $\varphi_{m}=\varphi_{g} \alpha_{g}+\varphi_{w}\left(1-\alpha_{g}\right)$, where $\varphi$ can be density, viscosity, etc. The density of gas conforms to the ideal gas law, and the density of water is assumed to be constant.

\section{B. Turbulence model}

The original $k-\varepsilon$ turbulent model has been used in this study. The turbulence kinetic energy, $k$, and its rate of dissipation, $\varepsilon$, are obtained from the following equations:

$$
\begin{gathered}
\frac{\partial}{\partial t}\left(\rho_{m} k\right)+\frac{\partial}{\partial x_{j}}\left(\rho_{m} k u_{j}\right)=\frac{\partial}{\partial x_{j}}\left[\left(\mu_{m}+\frac{\mu_{t}}{\sigma_{k}}\right) \frac{\partial k}{\partial x_{j}}\right]+G_{k}+G_{b}-\rho \varepsilon-Y_{M} \\
\frac{\partial}{\partial t}\left(\rho_{m} \varepsilon\right)+\frac{\partial}{\partial x_{j}}\left(\rho_{m} \varepsilon u_{j}\right)=\frac{\partial}{\partial x_{j}}\left[\left(\mu_{m}+\frac{\mu_{t}}{\sigma_{\varepsilon}}\right) \frac{\partial \varepsilon}{\partial x_{j}}\right]+C_{1 \varepsilon} \frac{\varepsilon}{k}\left(G_{k}+C_{3 \varepsilon} G_{b}\right)-C_{2 \varepsilon} \rho \frac{\varepsilon^{2}}{k}
\end{gathered}
$$

while $G_{k}$ represents the generation of turbulence kinetic energy due to the mean velocity gradients, $G_{b}$ is the generation of turbulence kinetic energy due to buoyancy, and $Y_{M}$ represents the contribution of the fluctuating dilatation in compressible turbulence to the overall dissipation rate. $C_{1 \varepsilon}=1.44$ and $C_{2 \varepsilon}=1.92$ are constants. $\sigma_{k}=1.0$, and $\sigma_{\varepsilon}=1.3$ are the turbulent Prandtl numbers for $k$ and $\varepsilon$, respectively. The turbulent viscosity is defined as:

$$
\mu_{t}=\frac{C_{\mu} \rho_{m} k^{2}}{\varepsilon}, \quad C_{\mu}=0.09
$$




\section{Computational details}

The computation is carried out in the domain as shown in Figure 1 because of symmetry of the flow field. The grid with 19200cells was used in numerical simulation, and a finer grid is prescribed in the nozzle area and near the central line. For inlet, the stagnant temperature is $3000 \mathrm{~K}$, and the stagnant pressure is $5 \mathrm{MPa}$; for outlet, the pressure is $0.59 \mathrm{MPa}$. Reynolds number $5 \times 10^{5}$, is calculated based on the nozzle outlet diameter and local gas properties. Prandtl number of gas $P_{r, g}$ is 0.536 , and Prandtl number of water $P_{r, w}$ is 5.83. Thermal conductivity of gas and water are $0.486 \mathrm{~W} / \mathrm{m} \cdot \mathrm{K}$ and $0.613 \mathrm{~W} / \mathrm{m} \cdot \mathrm{K}$, respectively. Initially, the fluid in the domain is stationary: while the temperature is $300 \mathrm{~K}$, the pressure is $0.59 \mathrm{MPa}$, and the velocity at the nozzle inlet is $10 \mathrm{~m} / \mathrm{s}$.

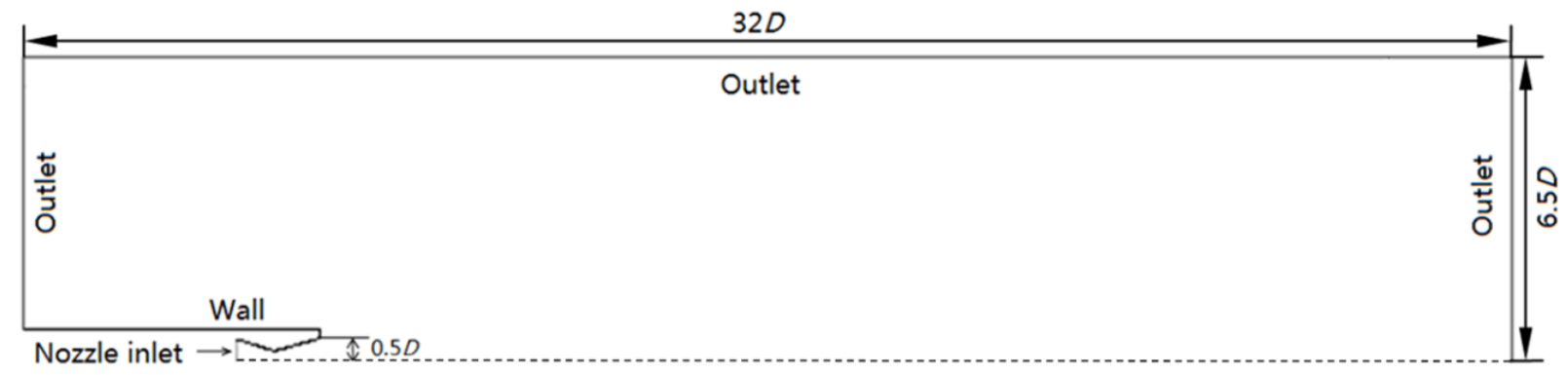

Axis of Symmetry

Figure 1. Boundary condition for gaseous jet injected into water

\section{Results and discussion}

The physical problem investigated in this paper is a supersonic gaseous jet injected into water from a bottomblowing mounted nozzle, where a converging-diverging Laval nozzle is used. The concerned flow field is the internal and external region of the nozzle. The nozzle is operated in the overexpansion condition. The design parameters are as follows: diameter of nozzle throat $d_{t}=0.04 \mathrm{~m}$; interior diameter of nozzle exit $D=0.08 \mathrm{~m}$; diameter of cross-section of propulsion system $d_{s}=0.11 \mathrm{~m}$; exit pressure $P=0.1485 \mathrm{MPa}$; exit velocity $U=1957 \mathrm{~m} / \mathrm{s}$; ratio of specific heats of gas $\gamma=1.4 ; R_{g}=287.6 \mathrm{~m}^{2} / \mathrm{s}^{2} \mathrm{~K}$; atmosphere pressure $p_{1}=0.1 \mathrm{MPa}$; depth of working location $H=50 \mathrm{~m}$. The total thrust of the underwater propulsion system is calculated by the following formula $22,31,32$ :

$$
F=\dot{m} v_{e}+A_{e}\left(p_{e}-p_{a}\right)+\left(A_{s}-A_{e}\right)\left(p_{B}-p_{a}\right)
$$

The first term on the right side is the momentum thrust represented by the product of the propellant mass flow rate and its exhaust velocity. The second term represents the pressure thrust consisting of the product of the nozzle exit area and the pressure difference between the nozzle exit pressure and the ambient pressure. The third term represents the pressure thrust generated by the difference of the pressure on the rear and front surfaces. The schematic of the propulsion system, illustrating the method for computing the total thrust, is shown in Figure 2.

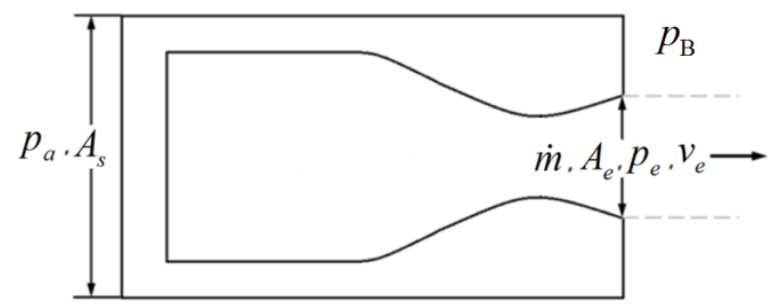

Figure 2. Schematic of the propulsion system, illustrating the method for computing thrust

\section{A. Jet process and flow structures}

Figure 3 shows the development of gaseous jet injected into water, where the results in column (1) are observed by Shi et al. ${ }^{18}$ via experimental methods, and the results in column (2)-(4) are obtained via the current Navier-Stokes flow computation. Although the experimental and numerical cases are based on different conditions, qualitatively 
similar flow structures are observed. The phenomena of expansion, necking/breaking, and back-attack ${ }^{27}$ have been highlighted, indicating that the flow development of gaseous jet injected into water is an unsteady process.

\section{Expansion process}

Initially, the inertia effect of the water will compress the gas behind the nozzle when gas jets enter water, which looks like a "gas bag" enclosed by the surrounding water ${ }^{18}$. When the gas is over-compressed so that the internal pressure is high enough, surrounding water can be pushed away, and the gas expands freely to complete the expansion process, as shown in Figure 3a. As is known, the initial transient is important during the entire propulsion period as the inertia effect of the surrounding medium might result in a failure of the engine. Recently, studies have focused on this aspect as well ${ }^{23,28,29}$. However, since the inertia effect of water is significant and persists, the later part of the expansion process is also important for underwater propulsion systems. As seen from the pressure distribution contour in Figure 3a, one can observe a low pressure zone behind the nozzle when the gas has fully expanded. The pressure difference on the rear and front surfaces of the propulsion system might generate a negative thrust.

\section{Necking/breaking}

At the end of the expansion process, gas expands too far causing the internal pressure to drop much lower than the ambient pressure. The "gas bag," shown in Figure 3a, will be compressed in the direction perpendicular to the flow. This principle is the same as that of the generation of a Mach disk. Sometimes the "gas bag" breaks into two parts, and sometimes it shrinks at the central part; this is called necking/breaking, and it is shown in Figure 3b. This phenomenon is the main factor that causes the process of a gas jet in water to become much more unsteady and turbulent than that of a gas jet in gas. Compared to the expansion process, the pressure behind the nozzle is much higher during the process of necking/breaking. A shockwave moves into the nozzle, and the velocity at the nozzle exit decreases. Necking/breaking makes the gas flow passage narrower or obstructive, as gas cannot flow forward and is compressed behind the nozzle to generate a high pressure. That is why the high pressure region appears before the break point.

\section{Experimental results Liquid volume fraction}
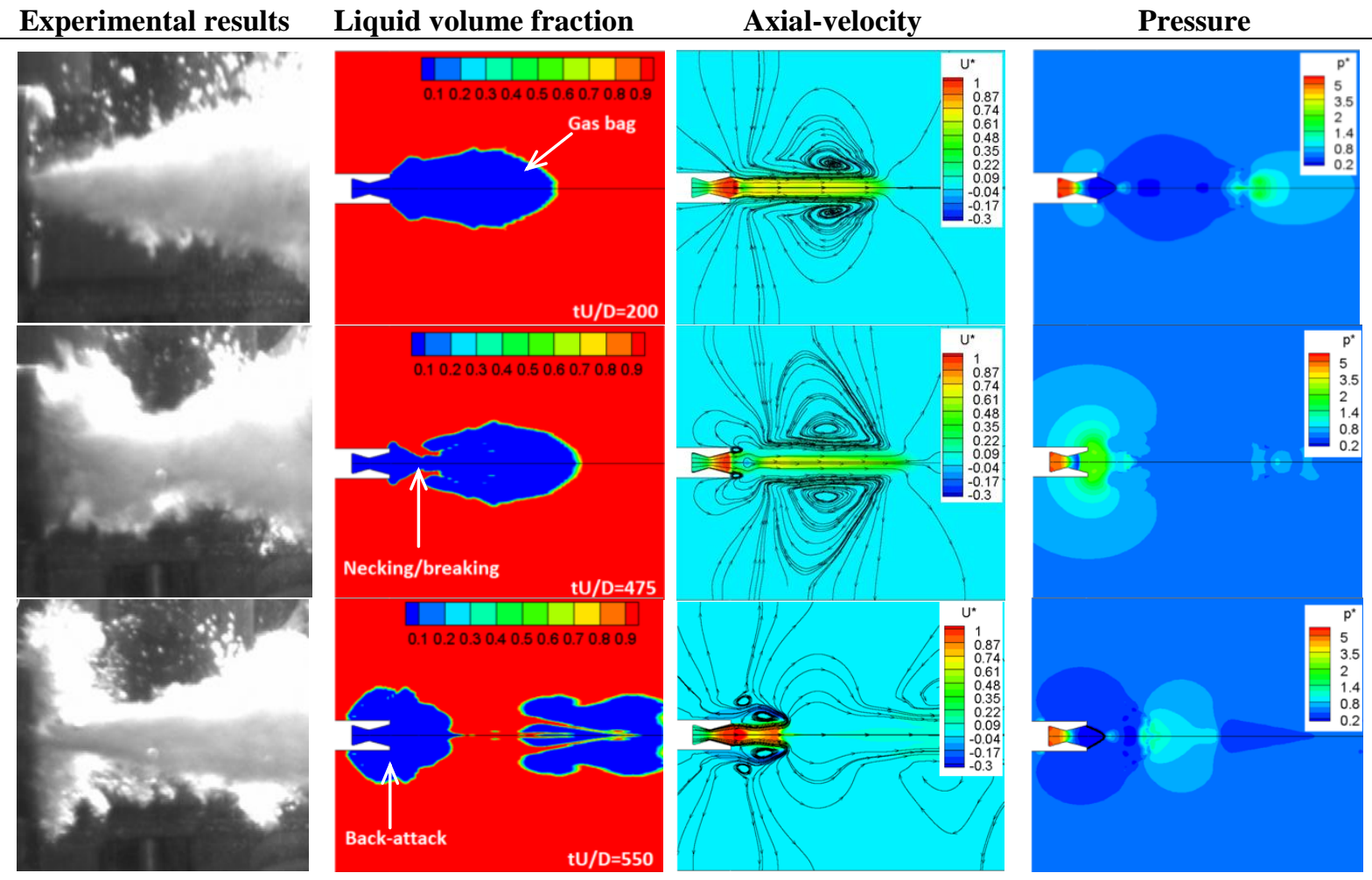

(a)
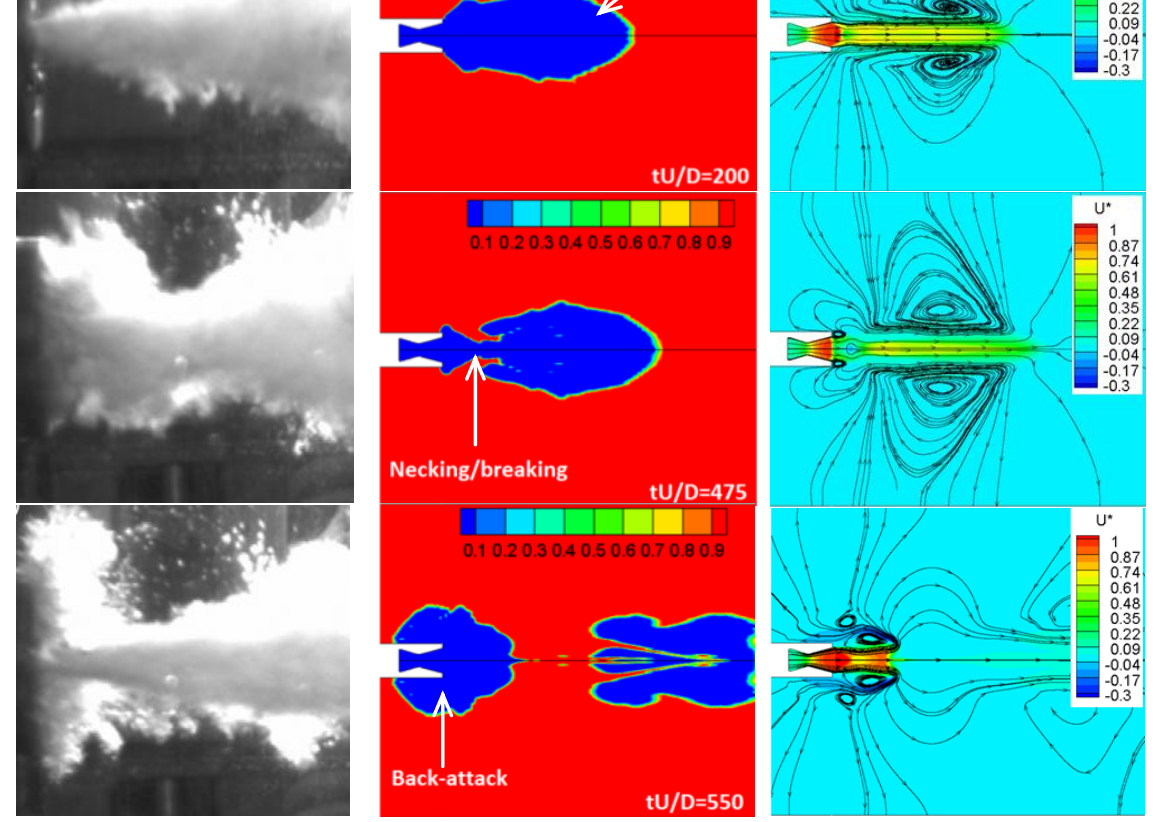

(b)

(c)

Figure 3. Flow structures of (a) expansion, (b) necking/breaking, and (c) back-attack process. Axial-velocity and pressure are normalized by $U^{*}=u / U, p^{*}=(p-P) / 0.5 \rho_{\text {ref }} U^{2}$ 


\section{Back-attack}

After necking/breaking, one part of the gas flows downstream while the other part reverses toward the nozzle. The reverse flow impacts the nozzle surface, and it can even enclose the end part of the propulsion system as shown in Figure 3c; this process is called "back-attack" 19, 27. A typical characteristic in the back-attack process is the presence of a negative axial-velocity. Aoki et al..$^{30}$ described that the major factor causing tuyere refractory erosion is the gas jet blowing backward to the tuyere and impacting on the front of the tuyere surface. They defined this jet blowing backward phenomenon as "back-attack" and found that it occurs after the jet necking. Shi et al. ${ }^{17,18}$ suggested that the back-attack is a shockwave feedback phenomenon. However, as seen from the axial-velocity contour and streamlines shown in Figure 3c, it is clear that the negative velocity only appears near and along the edges of the main flow passage, so that "back-attack" is not the whole gas jet blowing backward. Then, compared with the previous necking/breaking, the shockwave is moving out of the nozzle further indicating that back-attack is not a shockwave feedback phenomenon ${ }^{18}$. It appears that the underlying reason of back-attack is the block of passage. Since gas cannot flow forward after necking/breaking, it will turn to the surrounding side to form a circle flow causing the reverse flow in this region. The impact force generated in "back-attack" can result in the instability of underwater propulsion.

\section{B. Back pressure}

Back pressure, which is defined as the pressure exerted on the exhaust gas and against its flow direction, is an important factor in propulsion systems. The back pressure profiles acquired via Navier-Stokes computation and the bubble model for underwater gas jets have been compared and shown in Figure 4. Here, $p^{*}=(p-P) / 0.5 \rho_{\text {ref }} U^{2}$ and solution time, $t U / D$, are dimensionless parameters. $\rho_{\text {ref }}$ is the reference density at the nozzle exit. From the figure, it can be found that the two results are similar before $t U / D=50$. Beyond this moment, the pressure obtained via Navier-Stokes computation drops to a lower value, with the appearance of discontinued pulses. However, the pressure obtained by using the gas bubble model has no pulse, while it just fluctuates around the ambient pressure. To find out the relationship between the pressure and the flow structures, the 4 impulses are marked as numbers 1 through 4, which correspond to time equal to 475,750, 1250 and 1730. The corresponding volume fraction contours are shown in Figure 4. It can be observed that the pulses occur when the gas starts to break into two parts, where each pulse represents one time gas break. Current results are consistent with that presented by Wang et al. ${ }^{19}$. Overall, Navier-Stokes flow computation can describe gas break phenomena, so that back pressure pulses can be observed. However, in the gas bubble model, there is no back pressure pulse since gas is assumed to expand in the radial direction without breaking.

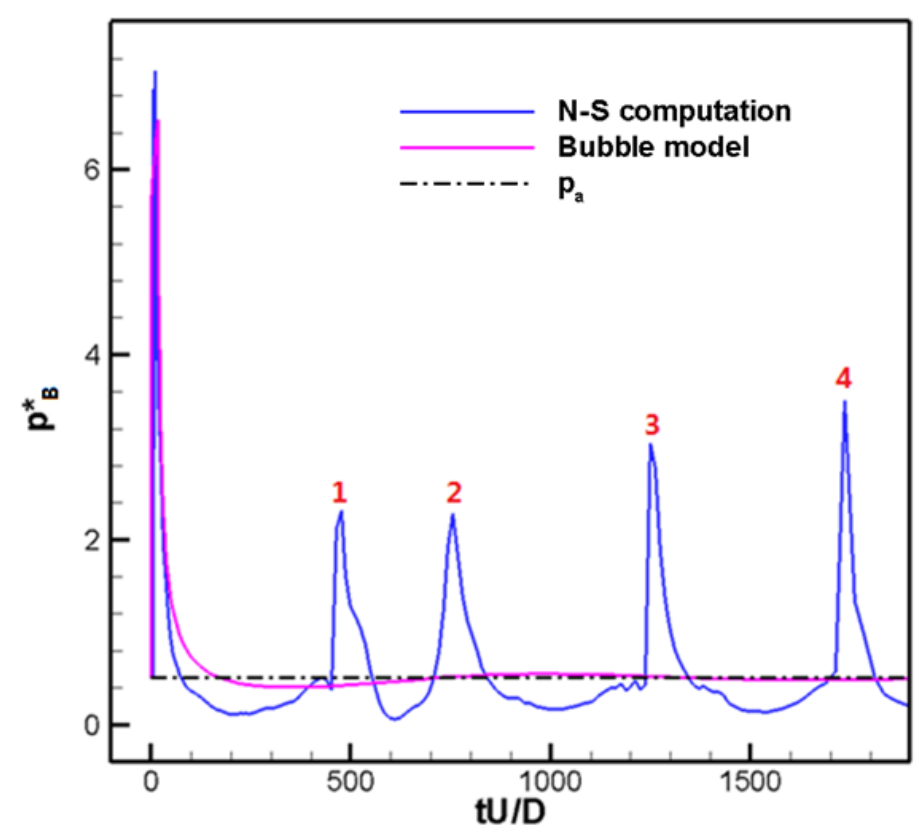

Figure 4. Back pressure acquired via bubble model and Navier-Stokes computation 


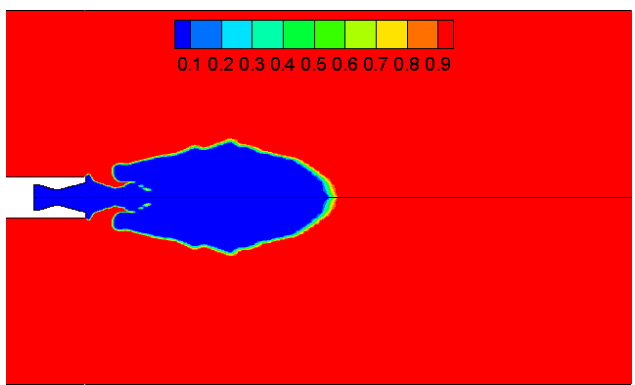

Pulse 1

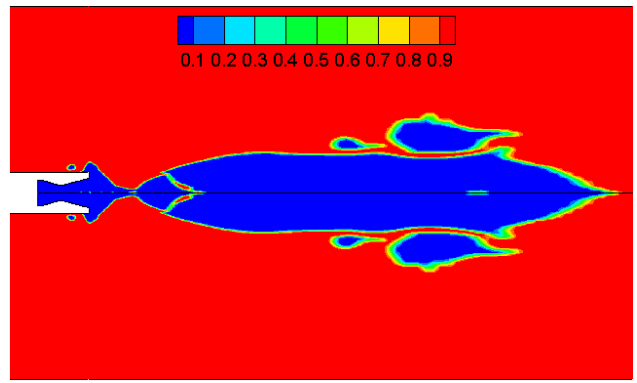

Pulse 3

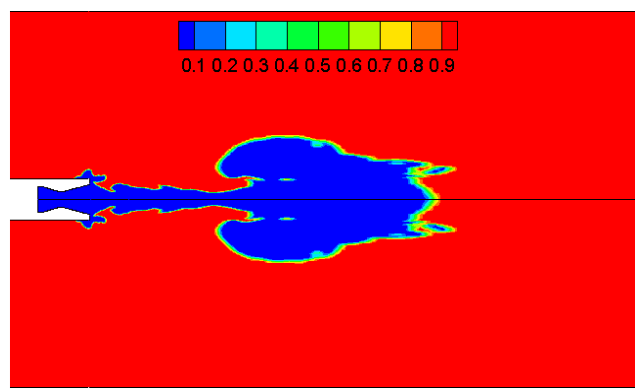

Pulse 2

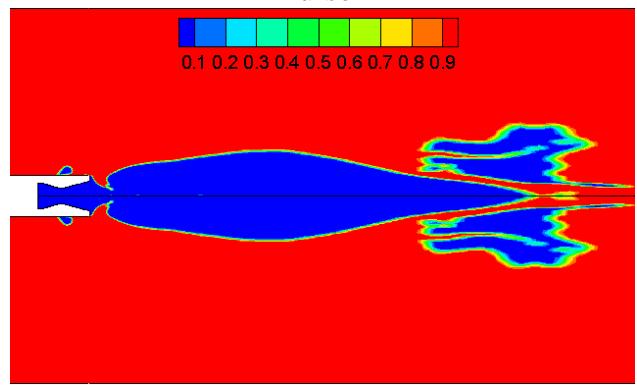

Pulse 4

Figure 5. Liquid volume fraction contour corresponding to the 4 pulses marked in figure 4.

\section{Normal velocity at nozzle exit}

A Laval nozzle is used in underwater propulsion systems. The gas is accelerated to supersonic speeds, and heat energy transfers to a high kinetic energy and generates thrust; thus, the normal velocity at the nozzle exit is another important factor for propulsion performance. Figure 6 shows the normal velocity profile at the nozzle exit obtained by using the bubble model and Navier-Stokes computation. It is clear that in the initial period $(t U / D<50)$, the two results are very similar; but in the later period, the result of the bubble model remains a stable value while negative velocity impulses occur in Navier-Stokes computation results. Comparing these 4 negative velocity pulses with the 4 positive pressure pulses shown in Figure 3, it is clear that both occur when time is equal to 475, 750, 1250 and 1730. Checking the pressure distribution and shockwaves corresponding to the 4 negative pulses, shown in Figure 7 , it is clear that the pressure around the nozzle exit is high because shockwaves have been pushed into the nozzle. Furthermore, the velocity in the bubble model is slightly higher than the maximum value in the Navier-Stokes computation results. One reason is that, unlike the bubble model, where the gas and water can be assumed to be inviscid, the Navier-Stokes computation considers the viscous loss in the nozzle area. Another reason is due to the difference in dimensionality of the two methods. The bubble model is one dimensional; the value of the normal velocity is equal to the velocity magnitude. However, in the two dimensional Navier-Stokes computation, the value of normal velocity is less than the velocity magnitude, since it only considers $\mathrm{x}$ component of the total velocity.

\section{Mass flow rate}

Besides the normal velocity at the nozzle exit, mass flow rate is another factor that affects the thrust. As seen from Figure 8, the mass flow rates calculated by using the two methods are similar, with a slight difference at the initial transient. As shown in the sub-figure, the mass flow rate of the bubble model continuously increases, while that of the Navier-Stokes computation fluctuates. Referring to the distribution of Mach number and shockwave complemented in Figure 9, it can be found that the shockwave is moving forward and backward in the nozzle from $t U / D=6$ to $t U / D=30$. When $t U / D=13$, the shockwave disappears because the back pressure is high enough to cause the velocity in the whole nozzle region to become subsonic. As a result, the mass flow rate decreases. After a while, since the velocity at the throat comes back to sonic, a shockwave appears again in the diverging part of the nozzle, and the mass flow rate increases to the maximum value. After that, both of the results keep the maximum value, because the back pressure is not high enough to push the shockwave to move into the nozzle throat. From this, it is clear that the mass flow rate is also affected by the back pressure. 


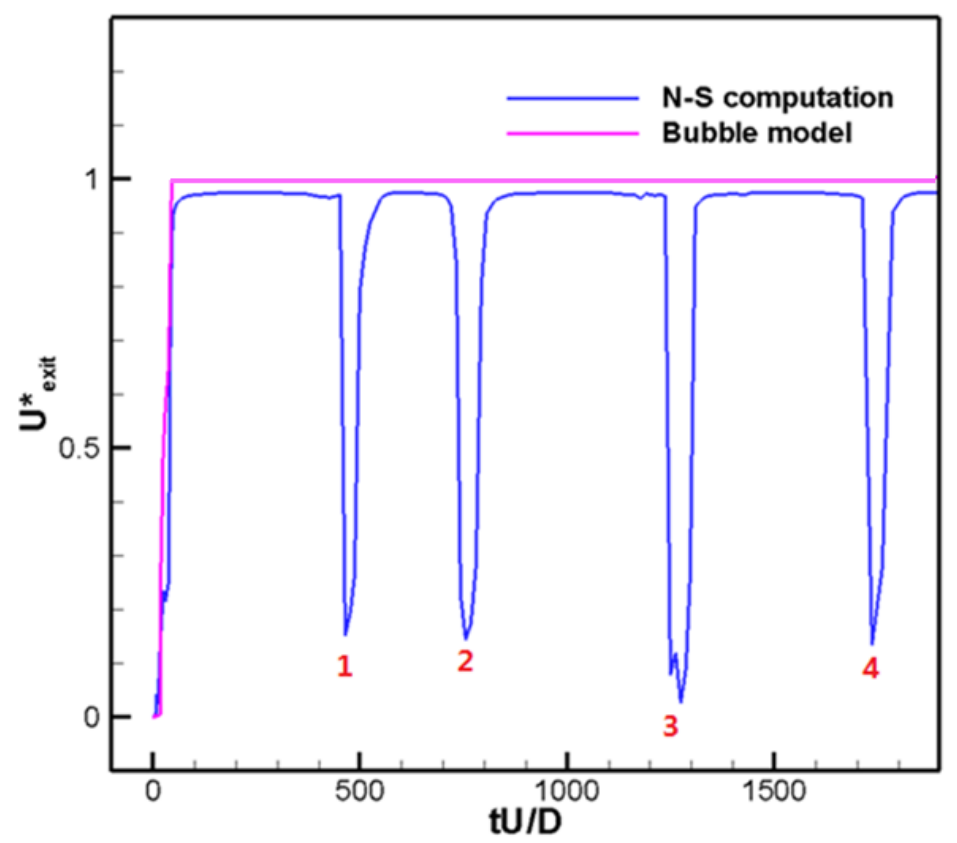

Figure 6. The normal velocity at nozzle exit acquired via bubble model and Navier-Stokes computation

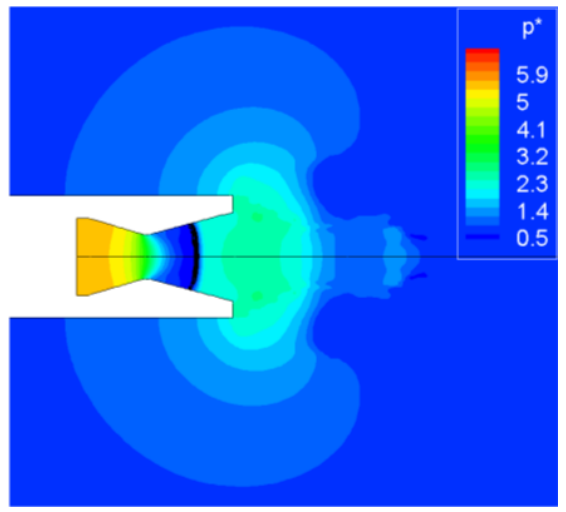

Pulse 1

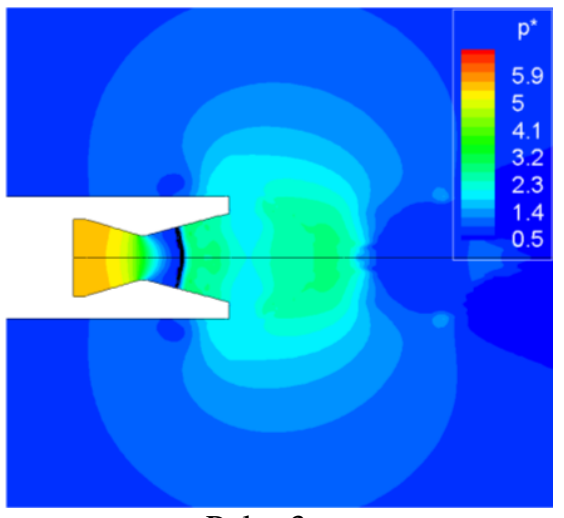

Pulse 3

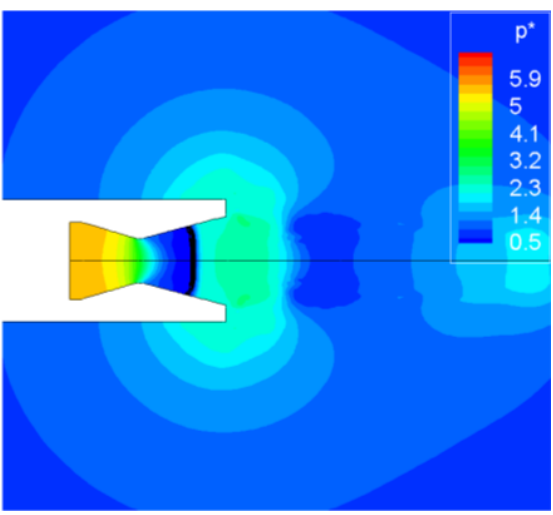

Pulse 2

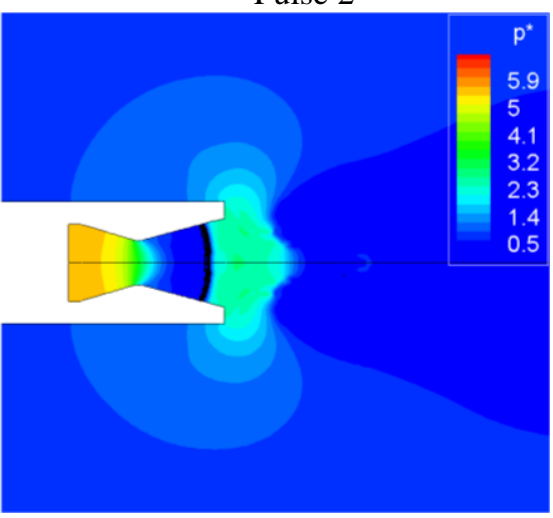

Pulse 4

Figure 7. Pressure distribution and shockwaves corresponding to the 4 pulses marked in figure 6 


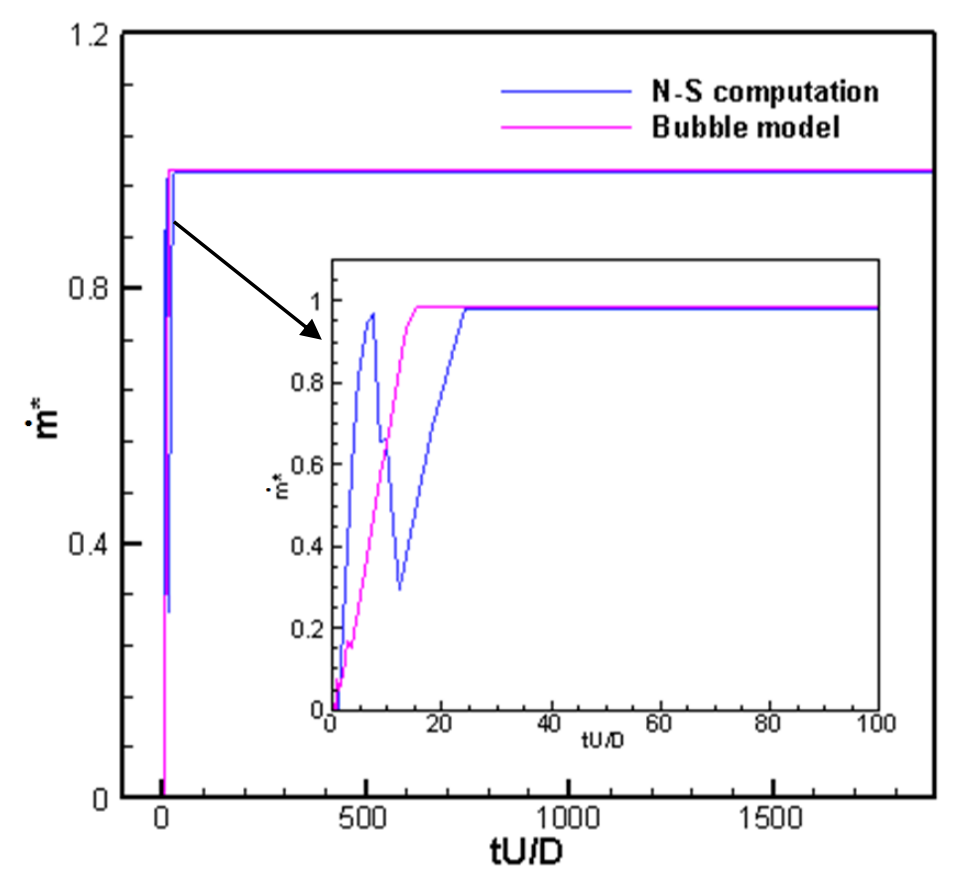

Figure 8. Time dependent mass flow rate acquired via bubble model and Navier-Stokes computation

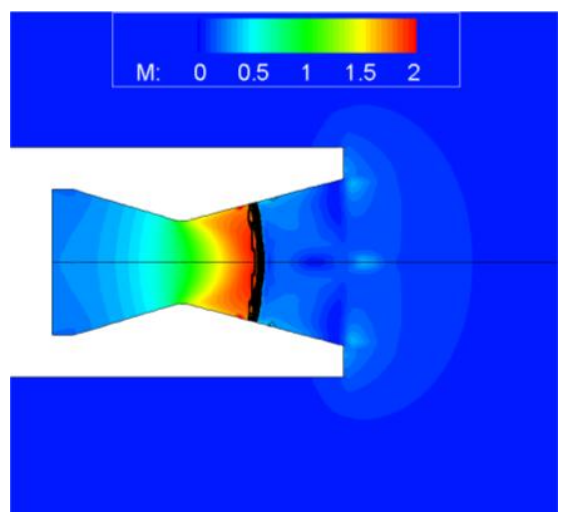

$t U / D=6$

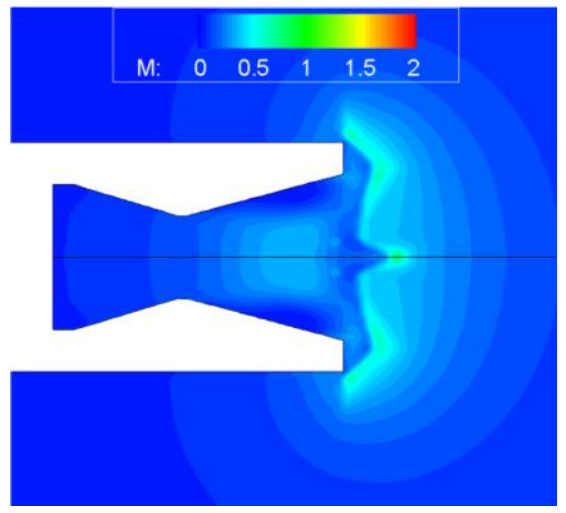

$t U / D=13$

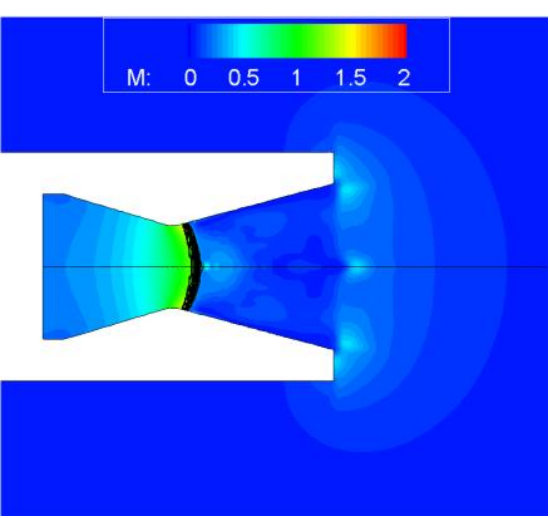

$t U / D=8$

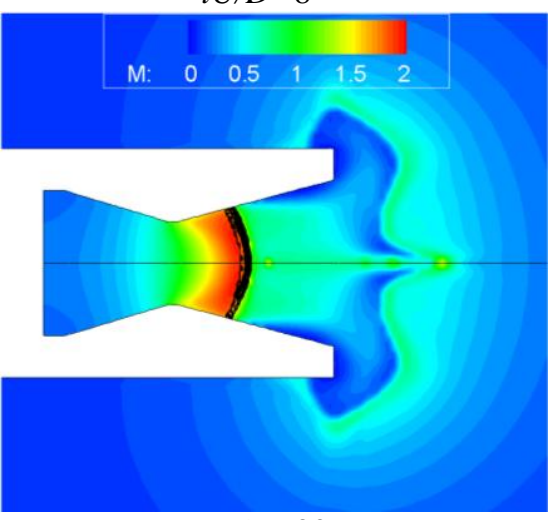

$t U / D=30$

Figure 9. The distribution of Mach number and shockwaves when $t U / D=6,8,13$ and 30 


\section{E. Pressure at nozzle exit}

Figure 10 shows the time dependent pressure profiles at the nozzle exit. The similar peak values of the two results appear at the initial transient. After that, the result of the bubble model decreases to the designed exit pressure which is determined by the nozzle geometry. But the result of the Navier-Stokes computation has pressure pulses which are similar to what appear in back pressure profile. The reason is that if the back pressure is lower than the critical value, the shockwave stays outside of the nozzle. The pressure at the nozzle exit is determined by the nozzle geometry. However, once the back pressure is higher than the critical value, the shockwave moves into the nozzle causing the pressure to become the back pressure. From above, it can be found that the pressure at the nozzle exit is not only determined by nozzle geometry but also by the back pressure.

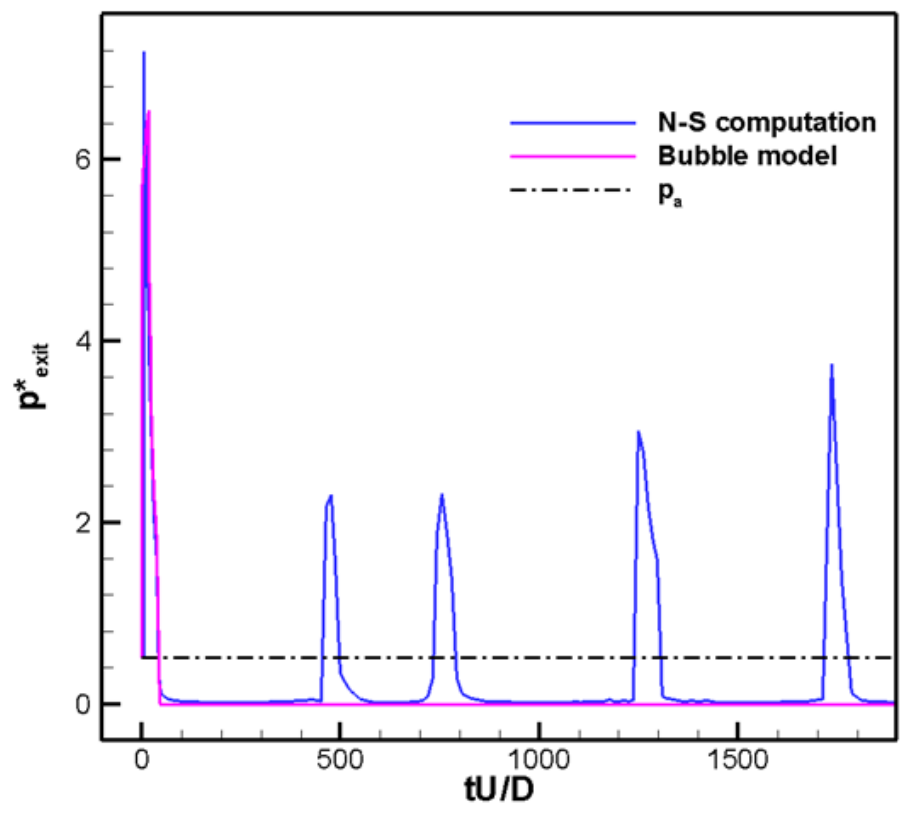

Figure 10. The pressure at nozzle exit acquired via bubble model and Navier-Stokes computation

\section{F. Thrust of underwater propulsion}

The total thrust of the underwater propulsion is calculated by Equation 11. The time dependent thrust profiles obtained via the bubble model and the Navier-Stokes computation are shown in Figure 11, normalized thrust $F^{*}=F / A_{e} P$. Initially, both of the results have a peak value. The research of Zhang $e t$ al. ${ }^{22}$, Shan $e t a l .{ }^{31}$, and Wang et $a l .32$ indicate that for underwater propulsion, the thrust exhibits a big jump at the initial stage as well. Beyond this stage, the thrust calculated by using the bubble model has no pulse, and it just fluctuates around a constant value slightly. However, the thrust predicted by the Navier-Stokes computation fluctuates with the appearance of discontinued pulses.

As shown in Equation 11, the total thrust is composed by 3 terms. The three components are analyzed based on the result of the Navier-Stokes flow computation, as shown in Figure 12. It is clear that the peak value of the total thrust is caused by the second term $A_{e}\left(p_{e}-p_{a}\right)$ and the third term $\left(A_{s}-A_{e}\right)\left(p_{B}-p_{a}\right)$. The negative pulses in $\dot{m} v_{e}$ are generated by the decrease in the nozzle exit velocity. The positive pulses in $A_{e}\left(p_{e}-p_{a}\right)$ and $\left(A_{s}-A_{e}\right)\left(p_{B}-p_{a}\right)$ result from the increase in the nozzle exit pressure and the back pressure, correspondingly. Overall, for underwater propulsion systems, the thrust fluctuates intensely. The discontinued pulses are caused due to the increase in the nozzle exit pressure and the back pressure which is influenced by the flow structures. 


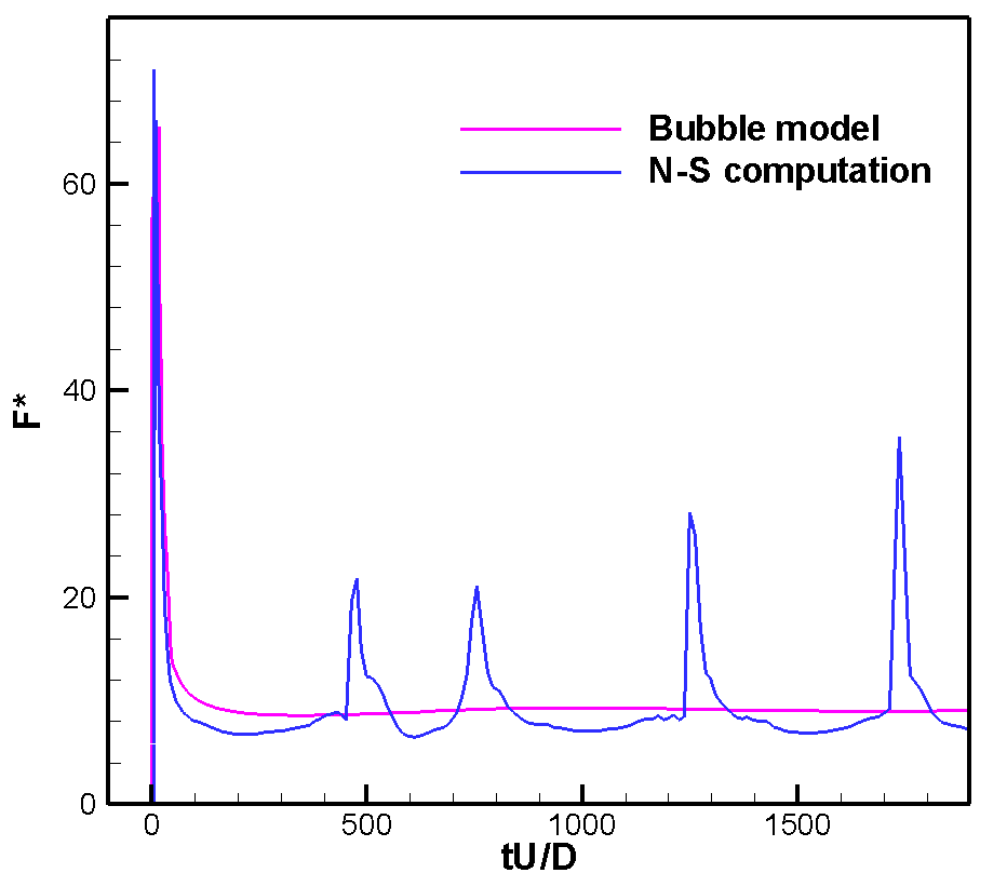

Figure 11. Total thrust of underwater propulsion system

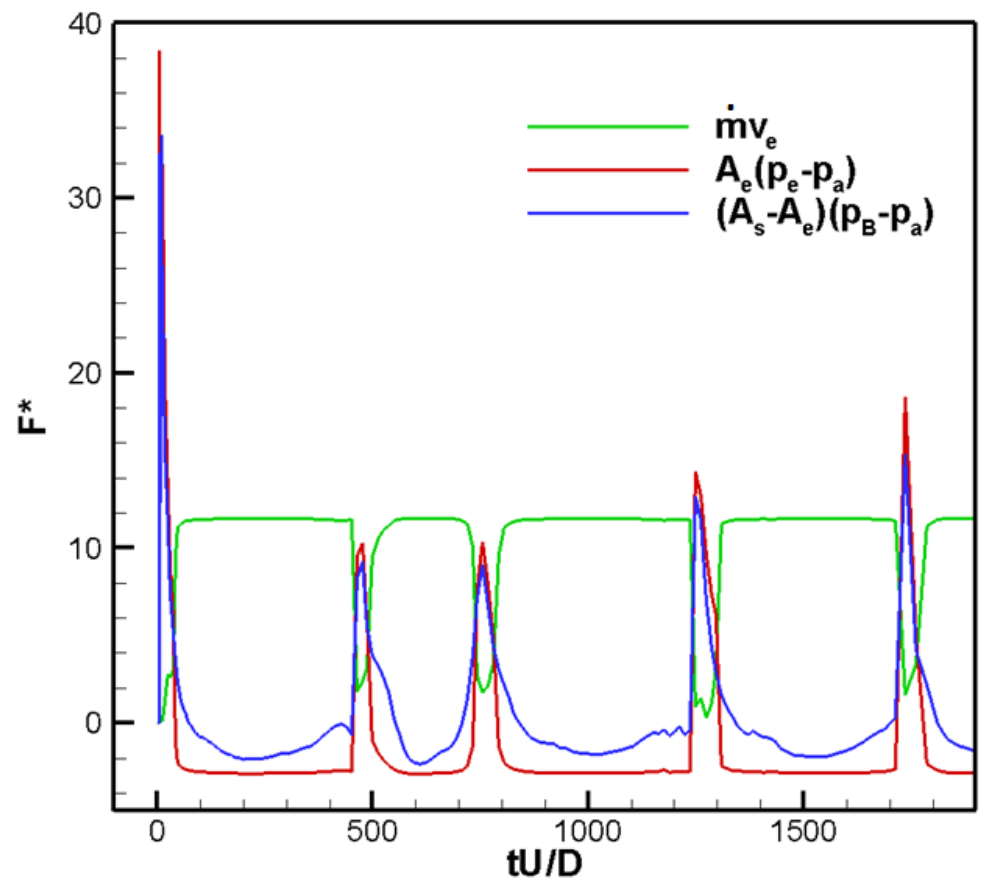

Figure 12. Three components of the total thrust which is predicted by Navier-Stokes computation

\section{Conclusion}

In the present work, both analytical gas bubble analysis and Navier-Stokes computations were used to elucidate flow structures of the gas-liquid interactions and to evaluate their effect on propulsion systems. The main findings are summarized below. The results indicate that: 
(1) The gas bubble model can produce a reasonable solution only for the initial transient process. Since the bubble model envisions continuing expansion in the radial direction with no break-up of the gas flow, it cannot predict the physical process after the initial stage of the flow development.

(2) The major flow characteristics observed in this study, including expansion, necking/breaking, and backattack are highlighted. In the gas expansion process, the gas pressure can drop to a level much lower than that of the ambient pressure, causing it to break up. Upon breaking up, the pressure before the break point increases due to the obstruction of the flow passage caused by the flow reversal. The flow reversal impacts the nozzle surface, creating the back-attack.

(3) The back pressure significantly affects the flow structures in the nozzle. As the back pressure elevates, shock can move into the nozzle, causing the exit velocity to become subsonic. A high back pressure reduces the mass flow rate as well as affects the difference of the pressure on rear and front surfaces.

(4) For underwater propulsion, the total thrust depends on the geometry of the system, the mass flow rate through the nozzle, the velocity and the pressure at the nozzle exit, the ambient pressure, and the back pressure which is influenced by the flow structures. The thrust fluctuates intensely with the appearance of discontinued pulses which are caused by the increase in the nozzle exit pressure and the back pressure.

\section{Acknowledgments}

The authors would like to acknowledge Mr. Chien-Chou Tseng, Mr. Chang-Kwon Kang, Dr. Hikaru Aono, Dr. Amit Gupta, Ms. Sabrina Marquina and Mr. DJ Lee for offering helpful discussions and comments.

\section{References}

${ }^{1}$ Chen, K., H. J.R., "Instability analysis of the transition from bubbling to jetting in a gas injected into a liquid," International Journal of Multiphase Flow, 1997, 23(4): 699-712.

${ }^{2}$ Longuet-Higgins M S, Kerman B R, Lunde K., "The release of air bubbles from an underwater nozzle," Journal of Fluid Mechanics, 1991, 230: 365-390

${ }^{3}$ He, X.Y., Ma, H.D., and Ji, C.Q., "Numerical simulation of gas jets in water,” Journal of Hydrodynamics, 2004, 19(2): $207-$ 212. (In Chinese)

${ }^{4}$ George A. Siamas, Xi, J., Luiz C. Wrobel, "Dynamics of annular gas-liquid two-phase swirling jets," International Journal of Multiphase Flow 35(2009): 450-467.

${ }^{5}$ Cao, J.Y., Lu, C.J., Li, J., and Chen, X., "Research on dynamic characteristics of underwater supersonic gas jets," Chinese Journal of Hydrodynamics 2009, 24(5):575-582. (In Chinese)

${ }^{6}$ Lindau, J.W., Venkateswaran, S., Kunz, R.F. and C.L. Merkle,. "Multiphase Computations for Underwater Propulsive Flows," AIAA 2003-4105.

${ }^{7}$ Surin, V.A., Evchenko, V.N., and Rubin, V.M., "Propagation of a gas jet in a liquid,” Journal of Engineering Physics, 1983 , 45: 1091-1101

${ }^{8}$ Koria, S. C., "Principles and applications of gas injection in steelmaking practice," Scandinavian Journal of Metallurgy. 22, 271. (1993)

${ }^{9}$ Qi, L. X., Cao, Y., and Wang, B.Y., "Experimental study of under-expanded sonic air jets in water," Acta Mechanica Sinica 2000 32(6): 667-675. (In Chinese)

${ }^{10}$ Loth E, Faeth G M. Structure of underexpanded round air jets submerged in water. International Journal of Multiphase Flow, 1989, 15(4): 589-603.

${ }^{11}$ Irie, T., Yasunobu, T., Kashimura, H., and Setoguchi, T., "Characteristics of the Mack Disk in the Underexpanded Jet in which the Back Pressure Continuously Changes with Time," Journal of Thermal Science, 2003, 12(2): $132-137$.

${ }^{12}$ Chang, K. S., Kim, J.K., "Numerical investigation of inviscid shock wave dynamics in an expansion tube," Shock Waves, 1995, 5: 33-45.

${ }^{13}$ Abate, G., Shyy, W., "Dynamic structure of confined shocks undergoing sudden expansion," Progress in Aerospace Sciences, 2002, 38: 23-42.

${ }^{14}$ Jiang, Z., Takayama, K., Babinsky, H., Meguro, T., "Transient shock wave flows in tubes with a sudden change in cross section," Shock Waves, 1997, 7: 151- 162.

${ }^{15}$ Liang, S. M., and Chen, H., "Numerical Simulation of Underwater Blast-Wave Focusing Using a High-Order Scheme," AIAA Journal, 1999, 37(8): 1010-1013.

${ }^{16}$ Dai, Z.Q., Wang, B.Y., Qi, L.X., and Shi, H. H., "Experimental study on hydrodynamic behaviors of high-speed gas jet in still water," Acta Mechanica Sinica 22(2006): 443-448.

${ }^{17}$ Shi, H.H., Wang, B.Y., Qi, L.X., “A submerged supersonic gas jet,” In: Proc. 7th National Congress on Hydrodynamics and 19th National Symposium on Hydrodynamics. Beijing: Ocean Press, 2005. 75-81. (In Chinese)

${ }^{18}$ Shi, H.H., Wang, B.Y., Dai, Z.Q., "Research on the mechanics of underwater supersonic gas jets," Science China, 2010 , 53(3):527-535. 
${ }^{19}$ Wang, B.Y., Dai, Z.Q., Qi, L.X., and Shi, H. H., "Experimental study on back-attack phenomenon in underwater supersonic gas jets," Acta Mechanica Sinica 2007 39(2): 267-272.

${ }^{20}$ Rogers, K.W., "A theoretical and experimental investigation of the transient phase of underwater rocket motor firing," University of Southern California Engineering Center Report, 1962.

${ }^{21}$ Richard J. Labotz, "Hydrodynamic Consideration and Limitations in Submerged Rocket Firings," Journal of Spacecraft and rocket, 1965, 2(3): 320-324.

${ }^{22}$ Wang, X.H., Chen, Y.L., Li, Q., Liu, C.J., "Nozzle flows of the launching under water,” Journal of Propulsion Technology, 2001, 22(1):61-64. (In Chinese)

${ }^{23}$ Zhang, Y.W., Wang, X.H., and Yang, J.X., "Study on working thrust for underwater engine using the spherical bubble model," Journal of Hydrodynamics, 2005, 20(5): 636-640. (In Chinese)

${ }^{24}$ Fabien Petipas, Jacques Massoni, Richard Saurel, "Diffuse interface model for high speed cavitating underwater systems," International Journal of Multiphase Flow 35(2009): 747-759.

${ }^{25}$ Yang, Q.X., Gustavsson, H., Burström, E., "Erosion of refractory during gas injection-a cavitation based model," Scandinavian Journal of Metallurgy, 1990, 19: 127-136

${ }^{26}$ Yang, Q.X., Gustavsson, H., "Effects of gas jet instability on refractory wear-a study by high-speed photography," Scandinavian Journal of Metallurgy, 1992, 21: 15-26

${ }^{27}$ Wei, J.H., Ma, J.C., Fan, Y.Y., "Back-attack phenomena of gas jets with submerged horizontally blowing and effects on erosion and water of refractory," ISIJ International, 1999, 39(8): 779-786

${ }^{28}$ Lu, C.J., Chen. F., Fan, H., Shen, X.H., "The fluid dynamic research on the underwater ignition," Acta Aeronautica et Astronautica Sinica, 1992, 13(4):b124-B130. (In Chinese)

${ }^{29}$ Ju, Y.T., Wu, X.X., Zhu, F.Y., "The study of air flow characteristic in nozzle and the thrust computation method in higher surrounding pressure," Journal of Ballistics, 2003, 15(3): 66-69. (In Chinese)

${ }^{30}$ Aoki T, Masuda S, Hatano A., " Characteristics of submerged gas jets and a new type bottom blowing tuyere," In: Injection Phenomena in Extraction and Refining. Wraith A E, ed. Newcastle: Department of Metallurgy and Engineering Materials (University of Newcastle upon Tyne), 1982. A1-A36

${ }^{31}$ Shan, X.S., Yang, R.G., Ye, Q.Y., "Fluid force on a vehicle with control system of vectorial thrust," Journal of Shanghai Jiaotong University, 2001, 35(4): 625-629. (In Chinese)

${ }^{32}$ Wang, C., Ye, Q.Y., He, Y.S., "Calculation of an exhausted gas cavity behind an under-water vehicle," Chinese Journal of Applied Mechanics, 1997, 14(3): 1-7. (In Chinese) 